\title{
MEMORIA Y SUBJETIVIDAD: UNA RELACIÓN DISCONTINUA ENTRE NARRATIVA Y TEMPORALIDAD PARA LA ENUNCIACIÓN DEL SUJETO COLECTIVO
}

\section{MEMORY AND SUBJECTIVITY: A BROKEN RELATIONSHIP BETWEEN NARRATI- VE AND TEMPORALLTY TO THE ENUNCIATION OF COLLECTIVE SUBJECT}

\section{MEMÓRIA E SUBJETIVIDADE: UMA RELAÇ̃̃o DESCONTíNUA ENTRE A NARRA- TIVA E TEMPORALIDADE, PARA A ENUNCIAÇ̃̃o DO SUJETTO COLETIVO}

\section{Páginas Alejandro Sepúlveda}

46-58 oliveriogiro@gmail.com

Recibido

15 de septiembre de 2015

\section{Aceptado}

2 de noviembre de 2015
Comunicador social-periodista. Especialista en comunicación educativa. Magistrante en Estudios Sociales de la Universidad Pedagógica Nacional en la línea de memoria, identidades y actores sociales. Docente del programa de Comunicación Social-Periodismo de la Facultad de Ciencias de la Comunicación, UNIMINUTO, sede principal. Director del Observatorio de Comunicación, Educación y Medios, UNIMINUTO.

Este artículo hace parte de los resultados parciales de investigación del Observatorio de Comunicación, Educación y Medios (OCEM) de la IV Convocatoria para el Desarrollo y Fortalecimiento de la Investigación en UNIMINUTO, 2015. 
Resumen

La relación memoria, narrativa y temporalidad para la construcción de subjetividades colectivas no se inscribe necesariamente en el tiempo cronológico de la modernidad. Las múltiples tramas que engranan la posibilidad de una narrativa que se alimenta de la relación entre historia y ficción, permiten la enunciación de las identidades que dan cuenta de una invención del pasado, desde la constitución de un yo, que narra y que está permeado por el juego subjetivo, para dar cuenta de una experiencia que se transmite a partir del relato. Para problematizar el lugar de estas relaciones, se acude a los adelantos del trabajo de investigación con el proceso organizativo Loma Sur, que tiene como intención revisar los acumulados históricos en sus prácticas edu-comunicativas y que permite establecer estas relaciones entre distintas temporalidades y la afirmación de unas identidades constitutivas del sujeto colectivo a partir de la memoria.

\section{Palabras clave}

Memoria, narrativa, temporalidad, subjetividades. 


\begin{abstract}
The relationship between memory, narrative and temporality to the collective subject construction does not necessarily remain in the in the chronological time of modernity. The multiple frames engaging the possibility of a narrative that feeds on the relationship between history and fiction allow the enunciation of identities that tell of a past invention, from the establishment of 'oneself', which relates and is permeated by the subjective game, to realize about an experience that is transmitted from the story. In order to problematize the place of these relationships, there should turn to the research progress with the organizational process Loma Sur, which intends to check the accumulated historical data in their edu-communicative practices and it allows establishing these relationships between different time frames and affirmation of constitutive identities of the collective subject from memory.
\end{abstract}

\section{Key words}

Memory, narrative, temporality, subjectivities

\section{Resumo}

A relação memória, narrativa e temporalidade para a construção de subjetividades coletivas não está inscrita necessariamente no tempo cronológico da modernidade. Os diferentes quadros que engrenam a possibilidade de uma narrativa que se alimenta da relação entre história e ficção, permitem a enunciação das identidades que dão conta de uma invenção do passado, partindo da constituição de um eu, que narra e que está permeado pelo jogo subjetivo, para dar conta de uma experiência que se transmite a partir do relato. Para problematizar o lugar dessas relações, nos voltamos para o progresso do trabalho de pesquisa com o processo de organização Loma Sur, o qual tem como objetivo revisar os acumulados históricos nas suas práticas edu-comunicativas e que permite estabelecer essas relações entre as diferentes temporalidades e a afirmação de umas identidades constitutivas do sujeito coletivo a partir da memória.

\section{Palavras-chave}

Memória, narrativa, temporalidade, subjetividades. 


\section{Una historia descentrada de la radio popular}

Es una tarde lluviosa de un sábado cualquiera del mes de julio del 2007, luego de reunirnos con varios de los jóvenes y niños del Comedor Comunitario Buenos Aires, con los que desde semanas antes veníamos preparando la primera emisión de "La onda del ocio", para la franja Loma Sur ${ }^{1}$ en la emisora comunitaria, tomamos un bus que en su aviso, destacaba con letras rojas "La Ye", "Altamira”, "Libertadores" y que desde lejos mostraba un personaje con medio cuerpo salido, que animaba el abordaje al vehículo, repitiendo los lugares por los que prometía pasar.

No sabíamos exactamente hasta donde íbamos, pero la indicación era preguntar dónde era "La Ye" y dos cuadras después bajarnos en el parque del barrio Nueva España, donde en algún lugar de esas calles empinadas del sur oriente, encontraríamos a los compas de Loma Sur que nos estarían esperando.

Durante el recorrido, algunos de los pelaos hablaban de sus nervios, de los posibles errores que cometeríamos, de las imprecisiones que habían quedado en el guion y que teníamos que corregir sobre la marcha. Compartieron la emoción de estar al aire y de saber que sus familias y amigos estarían pendientes de su incursión en la radio comunitaria. En ese momento no entendíamos muy bien a qué se refería esta especificidad. Llegar al lugar nos dio las primeras pistas, la emisora estaba ubicada en la tercera planta de una casa de familia, su fachada era como cualquier otra del sector, y la emisora como tal, se componía de dos consolas pequeñitas, dos parlantes colgados de manera cui-

1 Colectivo de comunicación popular de San Cristóbal, que inició los procesos de formación con emisoras escolares y organizaciones sociales en el sur oriente de Bogotá. Su nombre tiene que ver con la construcción de sentidos frente a la cultura popular de los barrios periféricos situados en los cerros del sur de la ciudad. dadosa en los extremos del cuarto, algunas sillas y una mesa donde estaban ubicados tres micrófonos que resaltaban por los filtros rojos de espuma.

Uno de los anfitriones nos saluda efusivamente y nos explica que la emisora comunitaria es nuestra palabra, que allí tenemos la oportunidad de contar nuestras historias, nuestros dolores y los sueños, que esta emisora es un esfuerzo de varias organizaciones y líderes comunitarios que quieren aportar a la construcción de formas dignas de vida para todos. Hasta ahí llegó el cuento, porque estábamos sobre el tiempo, por lo tanto no hubo espacio para ensayar, ni para arrepentirse, estábamos allí sentados, dándole inicio a una aventura sonora de la que no estábamos muy seguros. Un joven de unos 15 años manejaba la consola y con la mirada tranquila, de quien está curtido en la experiencia, dio la indicación para que hiciéramos silencio, de manera pausada dijo, "en 10 segundos estamos al aire", cruzamos miradas para ponernos de acuerdo en quien debería pronunciar la primera palabra y nos soltamos a conversar.

Mágica sensación, sentir que algunos de estos niños y jóvenes con los que venía compartiendo más o menos cuatro meses y que habían mostrado resistencia a la conversación, a compartir con otros, a relacionarse desde la palabra, ahora asumían de manera paradójica su voz para contar, para visibilizarse, para narrarse. El guion preparado se quedó a un lado, lo que creíamos era un programa sobre la cultura en la localidad, se convirtió en una conversación tímida sobre las cosas que nos gustan, los lugares que frecuentamos, la ocupación del ahora llamado tiempo libre, pero que representaba esos momentos sin tiempo y sin afán en los que consumimos los instantes de la vida para tratar de ser nosotros.

Poco a poco ese diálogo iniciado por dos o tres fue vinculando al resto del grupo, de tal manera que nadie se quedó sin hablar y así permanecimos durante una hora, impertinentes, desmesurados, con 
pequeñas pausas musicales y saludos permanentes a quienes nos imaginábamos estarían escuchando la emisión. Salimos carcajeando y todavía con los nervios de punta a tomar un pan con café y a conversar sobre la experiencia.

Odowen, como se hacía llamar uno de estos jóvenes que estaba seducido por el hip hop, se quejaba de su incapacidad para clarificar las ideas, para encontrar qué decir, para no sentirse bloqueado frente al micrófono. Hablaba de nuestros múltiples errores, las canciones que sonaron, lo que planeamos y nunca dijimos, los nervios, la voz temblorosa de todos, la incontrolable risa de los más pequeños. A pesar de los problemas, sabíamos en el fondo que había sido muy importante para todos y queríamos pensar que esta fuese la primera de muchas emisiones del programa y que la radio comunitaria nos permitiría consolidar un grupo juvenil en el Comedor Comunitario, que empezara a enfrentar las dificultades cotidianas con creatividad, entusiasmo y decisión.

Mucho tiempo después, pensando en aquel momento inaugural, creía que La onda del ocio, que no superó las cinco emisiones, fue muy importante para todos nosotros, porque allí encontrábamos una forma de enunciación que no entraba en nuestros códigos cotidianos, no porque la palabra fuera ajena a nuestras formas de relacionarnos cotidianamente, más bien entendiendo que el régimen discursivo ha privilegiado otro tipo de voces, en esas relaciones de saber poder, en donde se establecen las reglas de juego del decir, validando y deslegitimando unos relatos, temas, lenguajes y expresiones.

Habíamos escuchado programas de radio, narraciones de partidos de fútbol, música con locutores que exaltaban los artistas del momento, pero seguramente para ninguno de esos jóvenes, la idea de estar del otro lado del radio era una realidad posible y por eso es muy poderosa la idea de María Cristina Mata (2011), de afirmar la comunicación popular como el pronunciamiento de la palabra acallada, para hacerla audible, legitima como fuente de reconocimiento e interacción de los sectores populares, para la construcción de proyectos colectivos. Era pensar que aquellos que no se han contado, sino que los han narrado desde un lugar de poder, se disputan la posibilidad de proyectar sus relatos, desde formas de expresión que les habían sido negadas, pero que existen en su propia construcción de identidad.

De ese modo, la inquietud por la forma como los subalternos han recreado su cotidianidad, sus prácticas, formas de sobrevivir y mantener sus vínculos e identidades en contextos adversos, pasa por encontrar un lugar de enunciación que no está totalizado por la representación, sino por la construcción de solidaridades e identidades que se forman en y a través de la lucha (Yúdice, 2002).

Desde los años 80, en Bogotá se han planteado espacios de comunicación popular en el Sur Oriente, especialmente en el ámbito de los impresos, pero es solo hasta mediados de los noventa que la radio tiene su incursión, con la conformación de Voces Nuestras $^{2}$ y otras organizaciones en Usme, Ciudad Bolívar y Suba, que traían la idea de las emisoras comunitarias, sueño que unos años después se hizo realidad de manera clandestina y que funcionó regularmente para vincular procesos organizativos, para contar las historias cotidianas, para que las noticias enunciaran un contexto adverso, pero en el que la gente se plantea alternativas para seguir dando sentido a este territorio desde los seres humanos que lo crean y lo recrean todos los días.

La radio popular se ha constituido en Bogotá como una posibilidad de potenciar las luchas de los habitantes de las periferias, los altoparlantes, el ca-

2 Organización comunitaria de la localidad de San Cristóbal, que en los años 90 impulso los procesos de radio comunitaria, sin ser ni la primera, ni la única experiencia, pues en Usme ya se había dado rienda suelta a los noticieros comunitarios con altoparlantes y en la localidad de Suba también emergían por los años 90, procesos como Suba al Aire, hoy en día concesionario de la emisora con este mismo nombre. 
sete viajero y la radiocicleta, fueron sus primeras expresiones, luego la idea de emitir sin licencia en el dial, desafiando las prohibiciones del Ministerio de Comunicaciones, siendo perseguidos hasta bien entrados los primeros años del siglo XXI. De esta historia descentrada hace parte el proceso de la Red de Comunicadores y Comunicadoras de San Cristóbal, Loma Sur, que contribuyó en este sentido, como una apuesta por establecer desde el valor y la recuperación de la palabra, una posibilidad de transformar.

Esta es una historia descentrada porque se establece desde los rituales del encuentro, donde aquellos que hemos pasado por distintos momentos de este proceso social, hemos compartido un trozo del recuerdo para posicionarlo en la permanente disputa con relatos oficiales, totalizadores, ejemplarizantes que seguro dan cuenta de los procesos comunicativos en Bogotá desde otros lugares, experiencias y emocionalidades, pero que no constituyen para este colectivo un referente que permita ligar al pasado unas identidades, unas formas de explorar la subjetividad, que den cuenta de los acumulados políticos y educativos de la experiencia Loma Sur. Este tampoco es un relato definitivo, pues hablar de la memoria del proceso organizativo pasaría por entender desde dónde cuenta cada uno de los miembros, el valor que cada sujeto da a la experiencia, desde dónde se plantea el relato y las múltiples contradicciones que pasan por la narrativa que en últimas termina siendo constitutiva de un sujeto colectivo (Sarlo, 2005). Por tanto, la anterior resulta siendo una historia descentrada de la radio comunitaria, que acude a un momento "inaugural" de una experiencia cualquiera, posicionando algunos momentos, dejando de lado otros, que permite la enunciación de una subjetividad, dando cuenta de una explicación de lo que se es en el presente y que juega en la lógica de las discontinuidades temporales que permiten que la memoria sea invención ${ }^{3}$ del pasado que justifica un presente y la posición de sujetos que se proyectan a futuro.

3 “'Invención’ lo entiendo en el sentido de fabricación, de construcción histórica, como ha sido ampliamente utilizado en la teorización de la nación (Anderson[1983], 1991), de las identidades (Hall [1992], 2010) o del desarrollo (Escobar)"(Restrepo, 2013).
En ese sentido, ¿cómo se construyen las temporalidades, en los relatos del pasado del proceso organizativo Loma Sur? ¿Cómo se plantea una subjetividad colectiva a partir del relato histórico de esta organización social?

Estas son algunas de las preguntas de las que se trata de dar cuenta en este texto, no con el ánimo de generar respuestas que satisfagan las búsquedas de un lector situado en la larga trayectoria de los estudios sobre memoria, más bien se pretende problematizar el lugar de las relaciones entre tiempo, narración, testimonio y subjetividad, entendiendo que son múltiples los pliegues en los que se da la experiencia narrativa del tiempo, incluyendo su ficcionalización, el lugar del testimonio con sus posibilidades y limitaciones, para dar cuenta de los acontecimientos, el yo, que narra y que está permeado por el juego subjetivo de dar cuenta de una experiencia que se transmite a partir del relato.

\section{Discontinuidad y temporalidades ficcionadas}

Nuestra historia descentrada de la radio comunitaria, da cuenta de un acontecimiento de mediados del año 2007, al que aquí le hemos dado estatus de momento inaugural; sin embargo, Loma Sur, como proceso comunitario, inicia su trabajo en el 2002, como resultado de los encuentros ciudadanos en la localidad de San Cristóbal, en los que algunos de los participantes concluyen la necesidad de generar espacios comunicativos que den cuenta de problemáticas que no están necesariamente asociadas al tema del trabajo, los servicios públicos, la corrupción en la alcaldía local; más bien se pensaba que la cotidianidad, los espacios culturales y las expresiones de los pobladores de estos barrios, deberían tener un lugar, en la medida que se pudiera romper la contradicción de clase y plantear estructuralmente acciones de transformación con aquellos que no figuraban en los puestos públicos, pero que desde sus relatos tenían mucho por aportar. 
La idea de dar voz a los sin voz-luego cuestionada por el mismo proceso organizativo-, permitió empezar a generar redes de trabajo con profesores de los colegios públicos, organizaciones juveniles y culturales en la localidad, pensar en hacer un periódico, escarbar esas relaciones olvidadas con los impulsores de la emisora comunitaria y trabajar un proyecto político popular que vinculara a los vecinos desde la comunicación.

Lo curioso de este pasaje de la historia de Loma Sur es que cuando se empieza este proceso de investigación tratando de indagar por sus recorridos históricos, pocas veces sale a la luz este primer momento, las personas vinculadas a la organización generalmente han construido sus narraciones desde criterios distintos a las temporalidades cronológicas, sus relatos están dotados de emotividad, de acontecimientos significativos que hacen de la narración, un posicionamiento de la experiencia, para darle sentido al pasado. Para Beatriz Sarlo, "no hay testimonio sin experiencia, pero tampoco hay experiencia sin narración” (2005, p. 29), la experiencia cobra sentido en la medida que puede ser contada, que se pone en el lenguaje, en lo público, para liberarse del olvido.

De esa manera la narración está anclada al cuerpo, a una subjetividad que hace posible el recuerdo, pero este recuerdo no es una totalidad, pasa por el posicionamiento del sujeto, aspecto que desarrollaré más adelante; pero también la narración está inscrita en un tiempo determinado que no es el tiempo de la experiencia, sino el tiempo del recuerdo y de esta manera la narración está condicionada por el testimonio (Sarlo, 2005).

Esto hace que la temporalidad varíe con frecuencia, que permita la activación de los silencios, de privilegiar ciertos acontecimientos y no otros, que la misma construcción narrativa del testimoniante navegue en diversas temporalidades que permitan una elaboración ficcionada.
En ese sentido, el carácter de la experiencia ficticia del tiempo (Ricoeur, 1995), puede posicionarse en las memorias colectivas de Loma Sur. Pasar de un acontecimiento de un pasado relativamente cercano a otro que permite dar explicación a éste, sin tener en cuenta una temporalidad cronológica $o$ lineal, permite entender el valor que cobra el testimonio en su aparente falta de racionalidad y rigurosidad, con respecto a la disciplina histórica, para sumergirse en las apuestas de una subjetividad que no se pregunta por lo que pasó en realidad, sino por las construcciones que hacen los grupos humanos con esas representaciones del pasado que elaboran y re-elaboran en cada relato y que son constitutivas de su identidad. Otro elemento importante que tienen estas narrativas desde las discontinuidades temporales es que imprimen un carácter psicológico al relato, allí donde el sentir aporta a la construcción narrativa, pero que trae implícita una relación con aquel que escucha o lee este relato. De este modo, el testimonio no da cuenta de quién está contando, sino de las afectaciones que puede tener la memoria en quien la recibe.

Odowen es un joven de unos 25 años, participante del Comedor Comunitario Buenos Aires, que hizo parte de la experiencia radiofónica de la Onda del ocio; al indagar con él sobre esa primera experiencia en la emisora comunitaria, el hace énfasis en su interés por "acercarme a los grandes del hip hop en San Cristóbal, sabía de la relación de personajes como El Poeta o Mario Cantor, con la emisora y por eso me interesé en ir" (C. Moreno, comunicación personal, 15 de septiembre de 2015). Su narración esta mediada por los procesos artísticos que en ese momento eran desconocidos para él, pero de los que quería hacer parte como aficionado a la música y como seguidor de estos artistas reconocidos en San Cristóbal.

Sin embargo, su experiencia no se limita a este interés, en otro momento de la entrevista, habla del cambio sustancial que tuvo después de participar del trabajo en la emisora: 
Antes del programa, Alejo pasó por el comedor y nos dijo a los chinos ${ }^{4}$ que si queríamos volver al colegio, que habían unos cupos para la noche, a mí me dio fue pena porque yo me quedé en quinto y hace resto no estudiaba, a mí me da mucha mamera ${ }^{5}$ el colegio y ese día hasta nos reímos[...]; después del programa me acuerdo que le dije que si me ayudaba a conseguir un cupo, que ahora sí quería estudiar, porque imagínese que tocaba hablar en radio y yo ni siquiera sabía leer bien el guion, ya no podía salir de cualquier forma, tocaba leer bien y saber qué era lo que íbamos a decir (C. Moreno, 2015).

El relato de Odowen, está signada por su propia experiencia, por las contradicciones que asumía con respecto a este proceso radiofónico, su posibilidad de impulsarse como artista hip hop en San Cristóbal y por la necesidad de pensar en cambios trascendentales para darse un lugar como sujeto. Su narración está encarnada en la experiencia, pero determinada por el tiempo del recuerdo, que se desliga de las nociones comunes del tiempo físico en relación con un tiempo psíquico y varía según la emocionalidad de un tiempo interior (Arfuch, 2002), pues desde allí explica quién es, cómo su paso por la organización le permitió ser otra persona y pensarse desde otro lugar la vida.

Lo interesante desde el punto de vista de los juegos temporales, en el relato de Odowen y de otros miembros de Loma Sur, en ejercicios de recuperación colectiva de la memoria y entrevistas, tiene que ver con la capacidad de la narración de explicar los acontecimientos desde una discontinuidad que hace posibles los diálogos entre pasado y presente, en la necesidad de justificar que el presente

4 Expresión utilizada por el entrevistado para referirse a los niños y jóvenes que participaban del espacio comunitario.

5 Se refiere a una actitud de pereza o aburrimiento. es el reflejo de lo que se fue en el pasado (Arfuch, 2002) y de entender que las formas narrativas de la memoria resultan tan azarosas y contingentes como los sujetos que las enuncian.

\section{El sujeto constituyente en las memorias de Loma Sur}

Para Benjamin, la posibilidad del relato muere con el shock que deja enmudecida la experiencia después de la primera guerra mundial, esto conlleva a la separación del cuerpo y la narración que le quita al relato su sentido. Sin embargo, Benjamin hace un llamado a reconocer la redención del pasado a partir de la memoria, que devolvería al pasado su subjetividad (Benjamin, 1973). Una búsqueda por esa memoria que revitaliza la posibilidad de un presente, pasa por esas miradas subjetivas de los acontecimientos, que en este caso en particular, juegan con temporalidades múltiples y versiones diferenciadas que dependen de quién es el narrador, pero también de cómo se inscribe el relato para dar cuenta desde la experiencia. Allí donde Benjamin ve una imposibilidad de traer el pasado, dadas las condiciones de la modernidad y la inmediatez de la información, hay toda una inexplorada trama subjetiva que recae en el problema de las ciencias sociales desde la década del 60 del siglo XX y que pone explícita la necesidad de recuperar al sujeto.

Sarlo da cuenta del giro subjetivo en el que, las ciencias sociales empiezan a interesarse por aquellas historias que no invocan las grandes transformaciones de las estructuras mundiales o que están determinando el rumbo de la macropolítica económica, sino en las historias particulares que resaltan las rebeliones cotidianas, de grupos sociales pequeños, diferenciados, con unas particularidades que hacen posible ese análisis desde los pliegues culturales de la práctica y la afirmación de la identidad; es el momento de las nuevas historias, de nuevos sujetos, que despiertan interés por el detalle de su cotidianidad, por revelar las historias ignoradas por las matrices de la disciplina histórica, que aho- 
ra cobran protagonismo por el carácter novelesco de su relato, pero también por estas nuevas formas de contar, que ponen al sujeto como punto de referencia para la construcción del relato a partir de su testimonio, las cartas, las oraciones, las costumbres (Sarlo, 2005).

Esta dimensión de lo subjetivo también trae impresa una forma de enunciación del sujeto, esa forma está materializada en los relatos en primera persona, en la capacidad de darle un lugar al punto de vista de quien narra su propia historia, con todos los colores, sentidos y matices que tiene la experiencia humana.

La necesidad de la memoria es un imperativo cuando el clamor por justicia y no repetición se alzan como una bandera donde las colectividades parecen ya estar de acuerdo en algo. La memoria se ha posicionado como instrumento jurídico y como forma de construcción del pasado, después de que las dictaduras militares en Latinoamérica dejaran suspendida la posibilidad de entender ese pasado y de encausar la justicia sobre los responsables ${ }^{6}$ (Sarlo, 2005).

Esto da un estatus especial al testimonio que, para Arfuch, es lo que permite dar forma al sujeto a partir de la configuración de las narrativas que dan cuenta de un yo, que elabora los relatos desde la mediación entre la historia y la ficción, como posibilidad de contar el mundo de la vida (Arfuch, 2002).

6 Esto tiene que ver con la idea de que, desde los setenta, la historia oral y el testimonio han ganado un lugar importante no solo en el campo de la literatura, sino también en las ciencias sociales, dando un lugar sobresaliente a la subjetividad y al testimonio como símbolos de la verdad. Para Sarlo (2005), esto tiende a generar una tensión en los procesos de memoria que por un lado privilegia el lugar de las víctimas, de los acontecimientos que se deben recordar para que no se repitan y por otro lado el testimonio como única posibilidad para entender lo que realmente pasó y al que se le ha dado status de verdad.
Este aspecto ha sido trabajado desde Ricoeur (1995),Barthes (1967) yWhite (1992), en los que hay un relativo consenso al señalar que tanto la historia como la ficción se nutren de los mismos procedimientos de ficcionalización. En especial Barthes ha destacado el lugar de "espectáculo" de las narraciones inscritas en el discurso de la historia, que pretendiendo dar cuenta de acontecimientos reales, responde a una "ilusión referencial” desde el uso de ciertos procedimientos de escritura, como el "efecto de realidad" que pasa por la introducción de detalles que no necesariamente son relevantes para la trama de la historia, pero que funcionan como marcadores de realidad.

Ricoeur, por su parte, define un tercer tiempo modelado por la narración, que es producto del entrecruzamiento entre la historia y la ficción, distinguiéndolo de un tiempo cronológico, lineal, de sucesión de acontecimientos de la cotidianidad y de un tiempo lingüístico (aquel que se despliega en el acto de la enunciación y que tiene un carácter intersubjetivo); el tercer tiempo es el que se produce en el relato, que permite una refiguración de la experiencia en el tiempo mismo de la narración y que supone un sujeto distinto al que vivió cierto acontecimiento. De este modo, historia y ficción comparten los mismos procedimientos narrativos, pero se distinguen por su carácter de hechos reales o productos de la invención y por el tratamiento de las fuentes y archivos (Arfuch, 2002).

En el caso de los relatos testimoniales de las personas vinculadas a Loma Sur, las elaboraciones de la narrativa pasan por los momentos destacados del proceso, las grandes acciones que se entienden como significativas. En la mayoría de los casos, y a pesar de establecer entrevistas individuales, los sujetos dan cuenta de versiones compartidas, coherentes con la narración elaborada, pero que se entienden como acuerdos colectivos de lo que es la memoria de ciertos pasajes de la historia organizativa, que permitieron la problematización del colectivo frente a algunas coyunturas a nivel local, impulsando articulaciones con otras organizaciones de la localidad o de la ciudad. 
Otro elemento en las narrativas de los sujetos Loma Sur, tiene que ver con los detalles que se destacan de algunos acontecimientos, tratando de vincular la coherencia del discurso con una veracidad en los hechos narrados, con una intencionalidad de posicionarse y dejar claridad en las justificaciones políticas que hicieron que se actuara de determinada manera en un momento específico. Allí la experiencia ha dado sentido al recuerdo y la voz narrativa se ve mediada por el tiempo del relato, dado que las elaboraciones de ese pasado, dan cuenta de una identidad colectiva, elaborada desde una explicación de lo que se es en el presente, otorgando una relación temporal que condiciona las narrativas.

Es curioso que cuando se habla de los sueños frustrados, acontecimientos como la pérdida de la sede, la salida de la emisora o la disolución de la red de emisoras escolares, los relatos no son tan compartidos como en el caso anterior; allí las narraciones varían entre aquellos que le otorgan un sentido político a estos momentos de crisis y otros que confieren la responsabilidad a personas o actos individuales que profundizaron las rupturas. Para otros, estos acontecimientos no tienen una condición de fractura histórica, pues las afectaciones que dichos acontecimientos tienen en el proceso, son mínimas.

Tal vez el momento que más ha calado en la memoria colectiva ${ }^{7}$ del proceso organizativo, es el de la ruptura con la emisora comunitaria Vientos Estéreo, en primer lugar por las implicaciones históricas y políticas que tuvo este desacuerdo entre las dos organizaciones y de manera menos determinante, las consecuencias prácticas para los proce-

7 La memoria colectiva (Halbwachs, 1968), Blondel (1928) es un proceso social de reconstrucción de un pasado vivido o significativo para un grupo o comunidad y está mediado por marcos sociales como el lenguaje, el tiempo o la cultura en la que se inscriben los recuerdos. Para otros autores como Bartlett (1932) Los recuerdos se dan desde una actitud afectiva, que permite elaborar los relatos a partir de esquemas. Y para Bruner (2000) lo que constituye el recuerdo es la narrativa. Estos marcos sociales determinan la estructura del relato para que éste sea inteligible a aquellos que lo escuchan o la leen. Dan una sensación de fijeza y coherencia, estabilidad y persistencia a la memoria (Mendoza 2005). sos que por aquel entonces desarrollaba la organización. No es objeto de este documento dar cuenta de aquel pasaje, pero como resumen resulta importante mencionar que cuando la apuesta de radio comunitaria trascendió del espacio clandestino a la de una licencia del Ministerio de Comunicacione —hoy MinTic-, en el 2009, Loma Sur siguió participando de manera activa en la producción de contenidos para el proceso que arrancaba con la emisora legalmente constituida. Sin embargo, las condiciones cambiaron en la medida que la emisora necesitaba un sostenimiento y las discusiones para fijar los aportes de las organizaciones comunitarias que tenían espacios en la parrilla de programación, no permitieron que se establecieran acuerdos.

De esa manera, los espacios radiales que Loma Sur asumía ya no pudieron desarrollarse, pues las cuotas fijadas por la junta directiva de Vientos Estéreo, eran imposibles de pagar para la organización. Esta situación generó gran debate entre otras organizaciones participantes y acompañantes del proceso, pero la gestión de Loma Sur para establecer espacios de discusión colectiva y ajustar las cuotas nunca prosperó, de esa manera los miembros de Loma Sur han dado explicaciones que giran en torno a "una censura de parte de los compañeros de Voces Nuestras, por los contenidos de nuestros programas” (G. Leal, comunicación personal, 21 de noviembre de 2015), dando un estatus político a las causas por las cuales se da la ruptura entre las dos organizaciones y por otro lado una mirada más autoreflexiva del problema, como en el caso de los jóvenes que hacían el programa Cuartafonía, que efectivamente enuncian la incapacidad de Loma Sur para establecer diálogos y la expresión explícita del inconformismo en los últimos programas, que alteró el carácter de la discusión. En ese sentido se entiende una postura más autocrítica de lo acontecido y una elaboración del relato que difiere de las construcciones del otro grupo que disponía explicaciones desde la negación de la emisora comunitaria a las críticas que, como proyecto comunicativo, se le hacían desde los espacios radiofónicos de Loma Sur. 
Estas diversas memorias, problematizadas a partir del ejercicio de investigación, dan cuenta de lo que Sarlo (2005) llama la incompletud de la memoria, destacando que no hay un pasado completo, ni hay la posibilidad de establecer una versión totalizante desde el recuerdo.La memoria está fragmentada y es una auto-representación del pasado, por tanto, se construye de manera imaginativa y se complejiza en la medida que se pone como parte de un recuerdo colectivo, con la confrontación de otras fuentes:

La experiencia puede estar puesta en la vivencia de un acontecimiento, pero necesita de la imaginación para ir más allá, para entender que no es posible contener completamente el acontecimiento, que se escapan detalles desde diversas perspectivas y esto le otorga al testimonio la característica de incompletud. De esta manera lo que aporta la narración es posibilidad de ver el relato oculto o excluido que no se ha posicionado y que tiene cualidades importantes en re-lecturas de los acontecimientos (Sarlo, 2005, p. 55).

Esta idea de la incompletud también cobra sentido en las memorias colectivas; en la medida en que no se establece un proceso ordenado para contar la memoria, las narrativas de Loma Sur se entienden como una forma de establecer recuerdos que dan posibilidad de dotar de identidad al colectivo, pero allí hay ciertos vacíos y contradicciones temporales que generan la sensación de contar una historia incomprensible o irrelevante.

Para dar sentido a esta experiencia es necesario brindar, desde la imaginación, un toque de exterioridad a eso que se piensa está en un lugar de proximidad con la vivencia significativa de otros y que para ser narrado, debería cobrar esa distancia que la hace interesante y reflexiva.
Desde esta perspectiva, ¿qué es lo que se narra desde las memorias colectivas de Loma Sur? ¿Qué sujetos se enuncian desde allí? ¿Cuáles son esas narrativas compartidas que hacen posible que la memoria, constituya unas identidades?

Se parte de la idea de que la problematización de las temporalidades cronológicas, en la narrativa de Loma Sur, está asociada a una construcción simbólica y de sentido de los relatos. La forma como se enuncia cada sujeto en el colectivo, da cuenta de su experiencia particular, pero esta experiencia se subordina al orden de una memoria colectiva que hace que estos relatos no sean autónomos y que estén en permanente interacción con la producción de un sujeto colectivo, otorgándole legitimidad desde las prácticas cotidianas en la organización. Así, uno de los elementos que sale a flote como prioritarios para destacar en espacios de interacción con otras organizaciones es el de darle sentido a la comunicación como factor determinante en las relaciones humanas. Una de las consignas de la experiencia Loma Sur, es la de establecer puntos de encuentro, que permitan la unidad entre las organizaciones sociales del sur oriente y la herramienta clave para la humanización de este proceso es la comunicación.

De este modo, el sujeto Loma Sur se enuncia en su búsqueda por generar articulaciones, relaciones diversas con otros sujetos sociales y de propiciar acciones políticas que den cuenta del complejo entramado que significa ser habitante del sur oriente, ${ }^{8}$ entendiendo que la unidad se propicia a través de los diálogos que se pueden establecer desde los escenarios comunes y de la coordinación de las resistencias para hacerle frente a las necesidades de su comunidad.

8 Denominación para los pobladores urbanos de la periferia sur oriental de Bogotá, lo que hoy se conoce como las localidades de san Cristóbal y Usme. Zona caracterizada por albergar desde los años 50 a diferentes grupos sociales de todo el país como consecuencia de la violencia política y la creciente industrialización de la ciudad. 
En ese sentido, la narrativa de los procesos históricos de Loma Sur está signada por el recuerdo de importantes procesos de lucha social organizativa en San Cristóbal, las condiciones en que se han sorteado situaciones difíciles como la "limpieza social" o la situación de los trabajadores en la localidad, siempre desde la identidad que otorga el esfuerzo por articular los procesos y la necesidad de asumirse colectivamente desde la indignación. Allí se disponen unas memorias que dan cuenta de una identidad, del reconocimiento de un yo colectivo, que asume distancia en su narrativa con otros que construyen sus búsquedas desde otros lugares.

\section{Conclusiones}

Las complejas tramas de la temporalidad y de la constitución de las identidades desde el sujeto y su narración, pasan por la determinación de una permanente discontinuidad de los relatos, poniendo en evidencia elementos en disputa del contenido de la disciplina histórica y la memoria. En el caso de las narraciones examinadas desde el proceso organizativo Loma Sur, se resalta el juego ficcional con el que se elaboran los relatos, permitiendo establecer las condiciones en las que la memoria está signada por distintas temporalidades, que marcan un lugar en la subjetividad de quien está narrando y su intencionalidad.

No se puede pasar por alto que el régimen discursivo que ha impuesto el testimonio desde la primera persona, posiciona unas formas del relato que dan cuenta de lo que se es en el presente, justificado a partir de las representaciones que se construyen del pasado, enmarcando en el giro subjetivo el lugar de la memoria, en la construcción de identidad.

Estas formas de la memoria colectiva, si bien son puestas en disputa desde la confrontación de otras fuentes, lo que permiten es la reafirmación de un sujeto que desde el pasado pretende dar una lectura de su acumulado histórico; en ese sentido, los relatos que se construyen desde el proceso Loma Sur, permiten indagar no necesariamente en lo que realmente sucedió, más bien las preguntas que implica el estatuto epistemológico de la memoria nos plantean la necesidad de entender qué tipo de sujeto se está narrando, a qué identidades y construcciones colectivas obedece y por último, ¿qué hacen los sujetos con las interpretaciones del pasado que construyen? 


\section{Referencias}

Arfuch, L. (2002).El espacio biográfico: dilemas de la subjetividad contemporánea. Buenos Aires: Fondo de Cultura Económica.

Bartleet, F. (1932). La memoria social en el pensamiento soviético en David Middleton y Derek Edwards, Compiladores, Memoria compartida la naturaleza social del recuerdo y el olvido. Paidós, Barcelona, Pag 221 a 243

Barthes, R. (1967). «Introducción al análisis estructural del relato», en AA.W., Análisis estructural del relato, Buenos Aires, Tiempo Contemporáneo, 1970, 9-43.

Benjamin, W. (1973). El narrador. Revista de Occidente, 129, 301-333.

Blondel, C. (1928). Introducción a la psicología colectiva, Buenos Aires: Troquel.

Bruner, J. (2000). Actos de significado. Más allá de la revolución cognitiva, Alianza Madrid

Halbwachs, M. (1968).La memoria colectiva. Zaragoza: Prensas universitarias de Zaragoza.

Mata, M. (2011).Comunicación popular: continuidades, transformaciones y desafíos. Revista Oficios terrestres, Vol 1 no 26, pag 2.

García, J. M. (2005). La forma narrativa de la memoria colectiva. Polis. Investigación y Análisis Sociopolítico y Psicosocial, 1(1), 9-30.

Restrepo, E. (2013). Etnización de la negridad: La invención de las 'comunidades negras' como grupo étnico en Colombia. Popayán: Universidad del Cauca.

Ricoeur, P. (1985). Autobiografía intelectual, Buenos aires, Nueva Visión.
Ricoeur, P. (1995). “Experiencia ficticia del tiempo” en Tiempo y narración II. Buenos Aires:Paidos.

Sarlo, B. (2005). Tiempo Pasado. Cultura de la memoria y giro subjetivo. Una discusión. Siglo XXI.

Yúdice, G. (2002).Testimonio y concientización. En J. Beverley, y H.Achugar (Eds.),La voz del otro: testimonio, subalternidad y verdad narrativa. (p. 211-232). Guatemala: Universidad Rafael Landívar.

White, H. (1992). Metahistoria. La imaginación histórica en la Europa del sigloXIX. México FCE

. (1963). El contenido de la forma. Barcelona. Paidós 